\title{
Éléments de compréhension du processus de politisation du discours sur la gestion intégrée des zones côtières
}

The politicization of discourses on integrated coastal zone management

\section{Céline Ségalini}

\section{(2) OpenEdition}

Journals

Édition électronique

URL : http://journals.openedition.org/developpementdurable/9123

DOI : 10.4000/developpementdurable.9123

ISSN : 1772-9971

Éditeur

Association DD\&T

Référence électronique

Céline Ségalini, «Éléments de compréhension du processus de politisation du discours sur la gestion intégrée des zones côtières », Développement durable et territoires [En ligne], Vol. 2, n 3 | Décembre 2011, mis en ligne le 03 décembre 2011, consulté le 19 avril 2019. URL : http://

journals.openedition.org/developpementdurable/9123; DOI : 10.4000/developpementdurable.9123

Ce document a été généré automatiquement le 19 avril 2019.

\section{(c) (†) (8)}

Développement Durable et Territoires est mis à disposition selon les termes de la licence Creative Commons Attribution - Pas d'Utilisation Commerciale 4.0 International. 


\title{
Éléments de compréhension du processus de politisation du discours sur la gestion intégrée des zones côtières
}

\author{
The politicization of discourses on integrated coastal zone management
}

\author{
Céline Ségalini
}

1 Depuis les années 1990, l'expression "gestion intégrée des zones côtières » (GIZC) ${ }^{1}$, tout comme l'ensemble de ses déclinaisons ${ }^{2}$, est venue gonfler la liste des acronymes qui peuplent le discours des politiques publiques. Le nombre de textes académiques ou institutionnels qui comportent dans leur titre l'expression "gestion intégrée des zones côtières » ou dont l'entrée, par un moteur de recherche, se fait sous ce libellé rend ainsi compte de la large diffusion de ce discours politique. Cette banalisation interpelle puisque le discours GIZC est souvent présenté par la littérature spécialisée de manière strictement fonctionnelle, comme s'il relevait de simples choix techniques. Si un certain nombre d'écrits critiques font déjà état du caractère normatif et non purement technique de ce discours (Billé, 2004 ; Meur-Férec, 2008 ; Mc Kenna, Cooper et O'Hagan, 2008), un retour sur son émergence politique peut être éclairant. Il ne s'agit pas là de mettre un terme au débat déjà ouvert sur l'ancrage théorique et intellectuel de ce discours mais de reprendre certaines voies déjà explorées tout en suggérant de nouvelles pistes de réflexion.

2 À travers une compréhension socio-historique du discours GIZC, mise en perspective autour de l'évolution du discours écologiste depuis les années 1970, cet article invite à porter un regard différent sur son émergence politique. Il attire l'attention sur l'évolution politique générale concernant le traitement des questions écologiques et montre l'impact que ce changement de perspective a pu avoir sur la banalisation du discours GIZC en matière de politique de gestion du littoral. Après avoir montré quels sont les mécanismes généralement retenus par la littérature spécialisée pour dépeindre le processus de politisation de ce discours, nous montrerons l'importance à tenir compte du cadre 
cognitif général dans lequel ce discours s'inscrit. Ainsi, la politisation du discours GIZC s'explique en partie par la reconnaissance sociale du développement durable au tournant des années 1980/1990, rompant ainsi avec l'écologisme critique qui dominait le discours social dans les années 1970.

De manière générale, ce travail s'inscrit dans une approche singulière des études portant sur les questions écologiques. Elle consiste à appréhender ces problèmes comme des problèmes «socio-écologiques» (Hajer, $1995: 18)$, reconnaissant leurs dynamiques sociopolitiques. Depuis quelques années, des travaux de ce type se sont multipliés (Hajer, 1995 ; Lascoumes, 1994; Latour, 1999; Zaccaï, 2002; Dryzek, 2005). Ces travaux assez hétérogènes reprennent chacun à leur manière les approches constructivistes développées dans le champ des études sociologiques, et par suite dans les sciences sociales en général. Ils ont en commun l'idée que l'engagement au monde des acteurs se fait à partir de représentations socialement construites. Ils interrogent la manière dont les acteurs font exister des théories, des institutions, des outils, des normes, etc. et remettent ainsi directement ou indirectement en question les approches dites « réalistes » de l'environnement qui postulent que la manière de poser les problèmes écologiques reflète purement l'état de l'environnement naturel. Approcher les questions écologiques comme des construits sociaux peut paraitre réducteur; pourtant, la démarche ne consiste pas tant à nier l'importance des problèmes écologiques qu'à interroger ce qui est souvent perçu comme évident voire nécessaire dans la manière de les aborder (Hajer, 1995 ; Dryzek, 2005).

\section{Les mécanismes de politisation du discours GIZC retenus dans la littérature}

4 La littérature consacrée à la GIZC distingue trois grands facteurs ayant participé, dès les années 1970, à la politisation de l'approche « intégrée » dans le domaine de la gestion des zones côtières: le changement d'approche dans les études sur le littoral, le rapprochement entre scientifiques et gestionnaires, et enfin, l'inscription sur l'agenda politique de cette question tant au niveau national qu'international. Elle reconnaît aussi que cette première phase de politisation n'a pas eu d'impact considérable puisqu'il faut attendre le début des années 1990 et la large diffusion de la «pensée durable " pour que le discours GIZC devienne incontournable en matière de politique de gestion du littoral, dans de nombreux pays du monde.

\subsection{L'activité d'une communauté épistémique-gestionnaire transnationale}

Dans sa thèse consacrée à l'étude d'initiatives GIZC à Madagascar et en France, Raphaël Billé (2004) rappelle que la dimension intégratrice en matière de gestion des zones côtières n'aurait pu être portée au niveau politique sans un renouvellement des études sur le littoral autour de l'approche systémique. Née dans les années d'après-guerre aux Etats-Unis, la systémique se structure tout d'abord dans deux champs d'étude, la cybernétique et la biologie, pour ensuite donner lieu à de nombreuses applications. Elle se diffuse dans d'autres domaines aussi variés que l'urbanisme, le management, l'écologie et notamment les études sur le littoral. Cette approche s'efforce ainsi de prendre en compte d'autres aspects jusqu'alors moins développés dans notre compréhension du monde: 
l'instabilité, l'ouverture, la fluctuation, la complexité, etc. (Donnadieu et al., 2003). Appliquée au littoral, elle vise la prise en compte des différentes dimensions des zones côtières, qu'elles soient écologiques, économiques, sociales ou politiques.

Le changement de perspective dans les études spécialisées sur les zones côtières n'aurait pas eu l'impact qu'il a eu dans la gestion du littoral s'il n'avait pas été nourri par des échanges entre les communautés de chercheurs et les gestionnaires. En effet, la fortune politique de l'approche systémique des zones côtières tient surtout à l'implication de chercheurs, comme ceux du Center for Marine Policy de l'Université de Delaware (USA), dans l'amélioration des politiques de gestion du littoral ; l'activité de recherche étant orientée vers la formulation de solutions à des problèmes identifiés. Raphaël Billé (2004) dans une lecture proche des recherches effectuées par Biliana Cicin-Sain et Robert W. Knetch (1998), montre que ces échanges ont notamment été très importants outreAtlantique. C'est aux Etats-Unis, avec le Coastal Zone Management Act (1972), que l'idée de gestion intégrée des zones côtières aurait initialement été formalisée. Alain Miossec (2001) rappelle justement que cette expérience américaine n'est pas unique. En France, par exemple, les problèmes concernant le développement des littoraux sont posés en termes identiques dans le rapport Piquard (1973) même s'il n'est pas vraiment encore question d'utiliser le terme GIZC.

7 La politisation de l'approche intégrée relative aux zones côtières dépasse largement le simple rôle joué par l'échelon national. En effet, les institutions internationales avec leurs cohortes d'experts ont participé, elles aussi, activement à l'inscription de ce discours sur l'agenda politique international. Pour Norbert Calderaro (1994) et Olivier Lozachmeur (2005), le Conseil de l'Europe est pionnier en la matière; en adoptant, dès 1973, la résolution 73-29 relative à la protection des zones côtières, celui-ci reconnaît le défaut de coordination entre les approches sectorielles au niveau du littoral et affirme la nécessité de gérer de manière "intégrée " les zones côtières. L'activité internationale dans ce domaine se poursuit avec l'adoption par l'Organisation de coopération et de développement économiques (OCDE) de la recommandation $n^{\circ} \mathrm{C}-76-161$ du 12 octobre 1976. Deux ans plus tard, la Commission européenne participe à son tour à l'internationalisation de la démarche en publiant un rapport intitulé L'aménagement intégré du littoral dans la Communauté européenne (Lozachmeur, 2005). Comme le suggère Sandrine Kott (2008) pour son analyse des politiques sociales, il est important de "dénationaliser " les politiques et de mettre en avant la circulation transnationale des idées qui participent à leur formalisation. Le discours GIZC reste ainsi suspendu aux rapports, aux articles, aux conférences d'une communauté épistémique-gestionnaire, entendue comme un réseau transnational de chercheurs, d'experts, d'acteurs politiques partageant un savoir commun, tourné vers l'amélioration de l'action publique.

\subsection{L'affiliation de la GIZC au développement durable, son avènement suite à la Conférence des Nations Unies sur l'environnement et le développement}

8 La littérature rappelle aussi que si les principes du discours GIZC sont posés dès le début des années 1970, il faut attendre les années 1990 pour que l'approche acquière une reconnaissance politique large. Ainsi, pendant près d'une vingtaine d'années, celle-ci fait l'objet d'un travail d'institutionnalisation « sous-terrain » avec la mobilisation de tout un ensemble d'experts des zones côtières (Kalaora et Charles, 2000 ; Billé, 2004) : tous ces 
spécialistes, en entretenant ce discours, le produisent, le diffusent et le légitiment. Toutefois, ce processus d'institutionnalisation ne prend de sens qu'avec une large reconnaissance de la problématique du développement durable qui cristallise l'approche « intégrée » (Denis et Hénocque, 2001; Cicin-Sain et Knecht, 1998 ; Billé, 2004 ; Lozachmeur, 2005; Kalaora et Charles, 2000 ; Belfiore, 2003). L'expression apparait notamment au chapitre 17 de l'Agenda $21^{3}$, sorte de programme d'action de la Déclaration de Rio (1992). De par son inscription formelle sur l'agenda international, le discours GIZC devient alors un objet politique légitime et digne d'intérêt (Kalaora et Charles, 2000). On comprend pourquoi la plupart des travaux présentent la GIZC comme la déclinaison du développement durable au niveau des zones côtières, voire dans un sens plus «normatif», pour reprendre les mots de Raphaël Billé (2004: 98), comme «l'approche privilégiée pour gérer durablement les littoraux».

Suite à la Conférence des Nations Unies sur l'environnement et le développement tenue à Rio (1992), le discours GIZC est maintes fois ré-approuvé au niveau international, avec la révision et l'amendement de la Convention de Barcelone pour la protection de la Méditerranée en $1995^{4}$ et lors du Sommet de la Terre de Johannesburg en 2002 (Lozachmeur, 2005). Dans ce contexte d'approbation générale, les guides méthodologiques internationaux sur la GIZC se sont multipliés. Bilian Cicin-Sain et Robert W. Knecht identifient quatre grandes institutions internationales qui ont joué un rôle important dans la diffusion de ce discours avec la publication, dès le début des années 1990, de guides de «bonnes pratiques ", sortes de manuels expliquant les principes et les outils de la GIZC : l'Organisation de coopération et de développement économiques (1991), la Banque Mondiale (1993), l'Union Internationale pour la Conservation de la Nature (1993) et le Programme des Nations Unies pour l'Environnement (1995). On peut ajouter à cette liste les guides diffusés plus tardivement par la Banque Inter-Américaine de développement (1998) et la Commission Européenne (1999) ainsi que les guides UNESCO $(1997$; 2001). Des programmes, principalement financés par les grandes institutions internationales, vont favoriser la diffusion de l'approche à travers le monde. En dépit de certaines réticences ${ }^{5}$, la plupart des pays côtiers souscrivent à la GIZC. Dès 1993, au lendemain de Rio, pas moins de 75 pays ont déjà mis en œuvre des programmes de gestion intégrée du littoral, soit 217 expériences au total. Selon Stefano Belfiore (2003), presqu'une dizaine d'années plus tard, en 2002, on compte 145 pays impliqués dans de tels programmes pour 698 initiatives. Selon l'auteur, les pays du sud ont été parmi les premiers à mettre en œuvre des projets labellisés «GIZC » via l'appui des bailleurs de fonds multilatéraux et bilatéraux, comme la Banque mondiale, la Banque asiatique de développement (BAD), l'Agence japonaise de coopération internationale (JICA), etc. C'est en effet dans le giron de l'aide au développement et donc des pays du Sud, que les principes du développement durable et ses variantes ont dans un premier temps trouvé leur expression (Leroy, 2010). Compte tenu de leur dépendance aux soutiens financiers extérieurs, ces pays deviennent bien souvent des zones d'expérimentation. Des initiatives ont aussi vu le jour dans les pays du Nord. Olivier Lozachmeur (2005) souligne que l'approche a largement été diffusée en Europe avec la Stratégie européenne de gestion intégrée des zones côtières (2000), dont les recommandations sont validées par le Conseil et le Parlement Européen (2002). L'activité de recherche s'est elle aussi déployée, les publications et les rencontres scientifiques se sont multipliées, des associations ont même été créées ${ }^{6}$, renforçant toujours un peu plus la légitimité de cette approche. 


\section{Mise en contexte du discours GIZC à l'aune des évolutions du débat écologique}

10 L'aperçu socio-historique qui vient d'être présenté est celui généralement dépeint dans la littérature sur la GIZC. Or une mise en perspective du discours GIZC à l'aune de l'évolution du traitement de la question écologique dans nos sociétés peut être intéressante pour comprendre la tardive consolidation politique du discours GIZC et relativiser la banalisation dont il est actuellement l'objet dans le domaine des politiques publiques.

\subsection{Quand l'écologie politique tient le haut du pavé face à l'écopolitique internationale}

11 Dans les années 1970, au moment où certains textes institutionnels ${ }^{7}$ reconnaissent la nécessité de mettre en place une approche intégrée en matière de gestion des zones côtières et plus largement pour le traitement de la question écologique, on assiste à une très forte remise en cause des normes et des valeurs de la société moderne et notamment des effets destructeurs des pratiques de l'homme sur l'environnement: peur du surnombre avec une croissance démographique qui remet au goût du jour des réflexions malthusiennes, pollutions industrielles ${ }^{8}$, incertitudes relatives à la crise énergétique, critiques de la dégradation des conditions socio-économiques, critiques des sciences et des techniques, etc. Évidemment, ces revendications ne sont pas nouvelles : elles se sont notamment développées de manière coextensive à l'essor de la société industrielle (Morin, $1992^{9}$; Descola, 2005) ${ }^{10}$. Ce qui est nouveau dans les critiques formulées en cette seconde moitié de XXe siècle, c'est leur massification et leur diffusion dans l'ensemble des sphères sociales et politiques, contribuant à la structuration politique du secteur de l'environnement naissant (Lascoumes, 1994).

12 A cette époque, on assiste en effet à un changement dans l'appréhension des questions écologiques; celles-ci ne sont plus appréhendées comme des problèmes locaux et ponctuels (Theys et Kalaora, 1992) mais comme des problèmes de société. La prise de conscience de la question écologique s'est construite notamment autour de controverses sur l'usage des pesticides (Carson, 1962) ${ }^{11}$ ou encore sur le nucléaire, dénonçant ainsi la croyance invétérée dans la science et la technique. Cette prise de conscience se structure aussi autour d'une remise en cause du processus de croissance qui accompagne les dégradations de l'environnement comme en témoigne la publication par le Club de Rome du retentissant rapport Meadows (1972) qui préconise une « croissance zéro » pour les pays riches ${ }^{12}$. Profondément ancrée dans le débat public, cette position fut alimentée par tout un ensemble d'écrits protéiformes (Jacob, 1995), remettant en cause les modes de consommation, le modèle de production technicien, etc. (Charbonneau, 1963; Illich, 1971 ; Moscovici, 1972 ; Ellul, 1977). Bien que ces ouvrages ne forment pas une doctrine politique cohérente structurée autour d'un discours stable et institutionnalisé, ils sont pour ainsi dire reliés les uns aux autres par un air de famille dont on peut discerner des points de ressemblance. Ils sont représentatifs d'une conception singulière de l'écologie pour laquelle la protection de l'environnement passe par une réorganisation profonde de

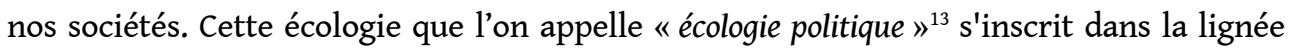
des critiques anthropocentristes pointées par l'écologie scientifique mais se veut plus radicale et insiste sur la nécessité de repenser les choix de société en dehors du 
développement de l'économie industrielle. Selon cette conception, le développement et la protection de l'environnement sont renvoyés dos à dos.

Tandis que dans les années 1970 le discours écologiste dominant se structure sur un ton très revendicatif et critique vis-à-vis des pratiques des sociétés modernes, le discours GIZC émergeant se construit quant à lui à l'image de l'écopolitique internationale ${ }^{14}$ encore marginale à cette époque (Lozachmeur, 2005). Plus complexe, le discours ainsi défendu au niveau international propose une conception qui ne remet pas directement en cause le développement porté par l'activité transformatrice de l'homme. Au lieu d'opposer la protection de l'environnement au développement, il est bien plus question d'intégrer les deux dimensions. Telle est notamment l'approche défendue par les instances onusiennes au chapitre 13 de la Déclaration finale de la Conférence des Nations Unies sur l'Environnement Humain, tenue à Stockholm en 1972: "Afin de rationaliser la gestion des ressources et ainsi d'améliorer l'environnement, les Etats devraient adopter une conception intégrée et coordonnée de leur planification du développement, de façon que leur développement soit compatible avec la nécessité de protéger et d'améliorer l'environnement dans l'intérêt de leur population $»^{15}$.

\subsection{Vers un déplacement du traitement de la question écologique}

Le discours écologiste de Stockholm, dont le discours GIZC est en quelque sorte l'expression, préfigure une conception de l'environnement qui se cristallise quelques années plus tard autour du discours sur le développement durable. Si la littérature ne manque pas de mettre l'accent sur l'affiliation du discours GIZC à celui du développement durable, elle omet de rappeler que c'est suite au déplacement des termes du débat sur l'environnement (Hajer, 1995 ; Dryzek, 2005 ; Semal, 2005) que ce discours s'impose. En effet, dès le tournant des années 1970/1980, les critiques portées par l'écologie politique s'essoufflent. Il est moins question de pesticides, de préservation des espaces dits " sauvages» ou encore de l'augmentation de la population mondiale (Theys et Kalaora, 1992 ; Hajer, 1995) : la conceptualisation proposée dans le rapport Brundtland (1987), nous apprend que les problèmes traités jusqu'alors de manière isolée doivent désormais être appréhendés ensemble, autrement dit "intégrés» (Dryzek, 2005; Semal, 2005; Rumpala, 2009). Si la philosophie est celle de l'intégration, on parle plus volontiers de « développement durable ». C'est avec la publication du rapport Brundtland en 1987, Our Common Future, que l'expression se constitue en étendard du discours écologiste dominant. A Rio (1992), la couverture médiatique de la conférence des Nations Unies pour l'environnement et le développement mais aussi le rassemblement de 178 pays, d'ONG, d'associations diverses, de représentants d'entreprises, vont avoir un impact considérable sur la diffusion du "discours durable» dans différentes sphères de la société. Le développement durable conquiert l'espace public général. Désormais, peu d'acteurs oseraient s'en passer pour légitimer leurs actions - même s'il reste largement obscur pour beaucoup d'entre eux (Smouts, 2005) et qu'il sous-tend une grande variété de significations et de projets (Dryzek, 2005 ; Rumpala, 2009). Sorte d'expression faitière, le développement durable porte dans son succès tout un ensemble d'expressions ("GIZC », " écosystème ", "biodiversité ", etc.) qui constituent autant de termes interconnectés (Jollivet, 2001) et plus ou moins interdépendants.

15 En quelques années, le « discours durable » et sa cohorte d'expressions se sont affirmés pour acquérir la force de l'évidence. Il tend à se naturaliser, si bien que la question de sa 
légitimité ne se pose plus. Pourtant, ces dernières années, depuis le tournant des années 2000, différents discours montrent les limites du développement durable, tant sur le plan environnemental que socio-économique. Une de ces critiques est portée par les objecteurs de croissance. Le mouvement qui prône la décroissance se structure autour de journaux comme Silence, Entropia, Ecorev', etc., de travaux d'auteurs (Latouche, 1994; Ariès, 2010). Dans le domaine spécifique de la GIZC, sans parler de décroissance, des critiques commencent aussi à s'élever. Dans leur article sur les risques d'érosion côtière, John Mc Kenna et ses collègues (Mc Kenna et al, 2008) dénoncent l'approche " intégratrice », telle que présentée par la Commission Européenne et plus précisément les aspects relatifs à la gouvernance. Ils montrent que dans une volonté de satisfaire tout le monde (logique du gagnant-gagnant) et en raison d'un manque de hiérarchisation des priorités, les principes de la GIZC se contredisent entre eux, empêchent d'établir une stratégie claire en matière de gestion du littoral et surtout conduisent à privilégier des intérêts particuliers de court terme. Toutefois, ces discours restent aujourd'hui minoritaires face à la prédominance du développement durable. On assiste ainsi à une " fermeture du discours» qui pour Marteen A. Hajer (1995) désamorce des débats plus fondamentaux relatifs aux liens entre société, technique et environnement.

\section{Conclusion}

L'analyse de l'émergence du discours GIZC comme discours spécialisé des politiques publiques montre que la politisation d'un discours est l'objet d'un long processus rythmé en partie par les représentations alors dominantes dans l'espace social. Pour le discours GIZC, le développement durable constitue cet " arrière-plan cognitif », en ce qu'il suscite une large adhésion dans les différentes sphères sociale, politique et économique. On comprend que le discours GIZC ne devient digne d'intérêt non pas seulement parce qu'il propose un mode de gestion du littoral porteur d'un savoir spécialisé et adapté mais aussi parce qu'il s'insère et participe du discours légitimé par un grand nombre d'acteurs.

Cette réflexion envisage donc de prendre du recul par rapport à un biais récurrent dans l'analyse des politiques publiques qui tend à étudier une politique publique pour ce qu'elle est, renforçant ainsi l' "hyperspécialisation» (Hassenteufel et Smith, 2002) au sein d'un domaine d'activité. Ce détour par la prise en compte du cadre global d'un discours politique spécifique, de sa porosité aux dynamiques sociales plus larges met l'accent sur l'importance qu'il y a à tenir compte de l'espace social dans lequel celui-ci se forme, relativisant ainsi son évidence.

\section{BIBLIOGRAPHIE}

Ariès P., 2010, La simplicité volontaire contre le mythe de l'abondance, Paris, La Découverte, 310 p. 
Belfiore S., 2003, « The growth of integrated coastal management and the role of indicators in integrated coastal management: introduction to the special issue ", Ocean and Coastal Management, $\mathrm{n}^{\circ} 46$, p. 225-234.

Billé R., 2004, La gestion intégrée du littoral se décrète-t-elle? Une analyse stratégique de la mise en œuvre, entre approche programme et cadre normatif, Paris, thèse soutenue le 29 octobre 2004 pour le grade de docteur de l'ENGREF, $473 \mathrm{p}$.

Calderaro N., 1994, « Droit et littoral en Europe », Études rurales, n¹33-134, p. 59-75.

Charbonneau B., 2002, Le Jardin de Babylone, Paris, Éditions de l'encyclopédie des nuisances.

Carson R., 1962, Printemps silencieux, Marseille, Editions Wildproject, 2009.

Cicin-Sain B. et Knecht R., 1998, Integrated Coastal and Ocean Management: concepts and practices, Washington D.C, Island Press.

Denis J. et Hénocque Y., 2001, Des outils et des hommes pour une gestion intégrée des zones côtières, Guide méthodologique, Volume II, UNESCO, 64 p.

Descola Ph., 2005, Par-delà nature et culture, Paris, Gallimard.

Donnadieu G., Durand D., Neel D., Nunez E., Saint-Paul L., 2003, « Diffusion de la pensée systémique ", in : L'approche systémique de quoi s'agit-il ? Synthèse de travaux du groupe AFSCET, http://www.afscet.asso.fr/SystemicApproach.pdf

Dryzek J-S., 2005, The politics of the Earth: environmental discourse, Oxford, New York, Oxford University Press.

Ellul J., 2004, Le système technicien, Paris, Le Cherche midi.

Greenger J. et Park T., 1994, « Political ecology », Journal of Political Ecology, n¹, p.1-12.

Hajer A.M., 1995, The Politics of Environmental Discourse. Ecological Modernization and the Policy Process, Oxford, Clarendon Press.

Hassenteufel P. et Smith A., 2002, « Essoufflement ou second souffle? L'analyse des politiques publiques “à la française" ", Revue française de science politique, Vol. 52, 2002/1, p. 53-73.

Hénocque Y. et Billé R., 2005, «Gestion Intégrée du Littoral : analyse des processus à l'œuvre et mesure des progrès accomplis dans le cadre de l'approche incrémentielle de l'intégration ", Colloque international « Prospective du littoral - Prospective pour le littoral : un littoral pour les générations futures », Ministère de l'Ecologie et du Développement Durable, Paris, p. 1-15. http:// www.iddri.org/Iddri/Equipe/gestionint-gr-eRB.pdf

Illich I., 1973, La convivialité, Paris, Éditions du Seuil.

Jacob J., 1995, « Les sources de l'écologie politique », Condé-sur-Noireau, Panoramiques-Corlet.

Jollivet M., 2001, Le développement durable, de l'utopie au concept : de nouveaux chantiers pour la recherche, Amsterdam, New York, Oxford, Paris, Elsevier.

Kalaora B. et Charles L., 2000, « Intervention sociologique et développement durable: le cas de la gestion intégrée des zones côtières », Natures Sciences Sociétés, Vol. 8, n², p. 31-38.

Kott S., 2008, « Une “communauté épistémique” du social ? Experts de l'OIT et internationalisation des politiques sociales dans l'entre-deux-guerres ", Genèses, Vol.2, n 71, p. 26-46.

Lascoumes P., 1994, L'éco-pouvoir : environnements et politiques, Paris, Éd. La Découverte. 
Latouche S., 1994, « Développement durable, un concept alibi », Revue Tiers-Monde, Vol.35, n¹37, p. 77-94.

Latour B., 1999, Politiques de la nature : comment faire entrer les sciences en démocratie, Paris, La Découverte.

Le Prestre Ph., 2005, Protection de l'environnement et relations internationales. Les défis de l'écopolitique mondiale, Paris, Armand Colin.

Leroy M., 2010, « Fondements critiques de l'analyse de la performance environnementale des dispositifs du développement durable », in : Palpacuer F., M. Leroy et N. Gérard (dir.), Management, mondialisation, écologie : regards critiques en science de gestion, Paris, Hermès science publications.

Lozachmeur O., 2005, « Le concept de "gestion intégrée des zones côtières" en droit international, communautaire et international », Droit Maritime Français, n657, p. 259- 277.

Marteen A., Hajer, 1995, The Politics of Environmental Discourse. Ecological Modernization and the Policy Process, Oxford, Clarendon Press.

Mc Kenna J., Cooper A. et O’Hagan A.M., 2008, « Managing by principles: A critical analysis of the European principles of Integrated Coastal Zone Management (ICZM) », Marine Policy, Vol. 32, $\mathrm{n}^{\circ} 6$, p. $941-955$.

Miossec A., 2001, «L'évolution de la géographie des océans et des littoraux face aux perspectives du développement durable au XXIème siècle. Quelles hypothèses envisager ? », Annales de Géographie, n621, p.509-526.

Meur-Férec C., 2008, De la dynamique naturelle à la gestion intégrée de l'espace littoral : un itinéraire de géographe, Edilivre, Collection Universitaire, Paris.

Morin E., 1992, « Pour une pensée écologisée », in : Theys J. et Kalaora B. (dir.), La Terre outragée. Les experts sont formels !, Paris, Éditions autrement, Série « Sciences en société », n¹, p. 66-77.

Moscovici S., 1976, La société contre nature, Paris, Union Générale d'Éditions.

Rodary E., Castellanet C. et Rossi G. (dir.), 2003, Conservation de la nature et développement. L'intégration impossible?, Paris, Karthala.

Rumpala Y., 2009, « Le développement durable comme reconstruction narrative d'un projet commun » in : Villalba B., Appropriations du développement durable. Émergences, diffusions, traductions, Villeneuve d'Ascq, Presses Universitaires du Septentrion, p. 39- 65.

Semal N., 2005, « Le développement durable est-il l'héritier du mouvement proenvironnement ?», Esprit critique, Vol.7, n¹, p. 100-115.

Smouts M-C. (dir.), 2005, Le développement durable : les termes du débat, Paris, Armand Colin. Theys J. et Kalaora B. (dir.), 1992, La Terre outragée. Les experts sont formels !, Paris, Éditions autrement, Série « Sciences en société ", $n^{\circ} 1$.

UNESCO, 1997, Guide méthodologique d'aide à la gestion intégrée de la zone côtière. Manuel et guides de la Commission Océanographique Intergouvernementale, n³6, 47 p. http://unesdoc.unesco.org/ images/0012/001212/121249fo.pdf

UNESCO, 2001, Des outils et des hommes pour une gestion intégrée des zones côtières, $\mathrm{n}^{\circ} 42,64 \mathrm{p}$. http:// unesdoc.unesco.org/images/0012/001245/124596fo.pdf 
Zaccaï E., 2002, Le développement durable. Dynamique et constitution d'un projet, Bruxelles, Bern, Berlin, Frankfurt/M, New York, Oxford, Vien, P.I.E. - Peter Lang S.A., Presses Interuniversitaires Européennes.

\section{NOTES}

1. La GIZC vise le développement durable des espaces côtiers. Il s'agit d'une approche holiste et intégrée dont on peut dégager différents principes de gestion : l'intégration sectorielle entre les secteurs d'activité afférents aux zones côtières, l'intégration écologique et économique, l'intégration des parties prenantes (acteurs économiques et politiques, usagers) aux différentes échelles (local, national, régional et international), l'intégration spatiale en reliant les domaines terrestre et maritime, l'intégration entre des logiques managériales et scientifiques (échange du savoir), l'intégration temporelle (démarche évolutive qui doit tenir compte des générations futures).

2. Dans les articles académiques ou les publications institutionnelles, il est tantôt fait référence à la "gestion intégrée du littoral » (GIL), à la «gestion intégrée des zones côtières » (GIZC), mais aussi à la " gestion intégrée des aires marines et côtières » (GIAMC), l'« aménagement intégré des zones côtières » (AIZC) ou encore la « gestion intégrée de la mer et du littoral » (GIML) depuis le Grenelle de la Mer. Cette liste n'est évidemment pas exhaustive, il semblerait que chaque institution propose sa formule. Cette diversité des labels se comprend comme le résultat de la multiplication des usages du discours GIZC et nous invite à se méfier de l'idée d'une définition unique et valable en toute circonstance. On peut toutefois parler d'une convergence dans la production des discours politiques. Aussi pour faciliter la lecture nous rassemblons sous le label GIZC l'ensemble de ces approches. Ce choix se justifie par le fait que le terme GIZC est celui qui officialise l'approche lors de la Conférence des Nations Unies sur l'Environnement et le Développement en 1992.

3. L'agenda 21, document sous-titré « Stratégie mondiale pour le développement durable » est en quelque sorte un programme d'action de la Déclaration de Rio. Le chapitre 17 de l'Agenda 21 s'intitule «Protection des océans et de toutes les mers, y compris les mers fermées et semifermées, et des zones côtières et protection, utilisation rationnelle et mise en valeur de leurs ressources biologiques ». Son sous-chapitre 17A qui fait directement référence à la GIZC a pour titre « Gestion intégrée et développement durable des zones côtières et marines, y compris de la zone économique exclusive ».

4. Voir sur ce point le protocole relatif à la Gestion Intégrée des Zones Côtières (GIZC) de la Méditerranée du 10 juin 1995 et qui complète la Convention de Barcelone pour la protection de la mer Méditerranée contre la pollution adoptée le 16 février 1976. Le mandat de ladite convention s'est élargi en vue d'inclure la planification et la gestion intégrée des zones côtières. http:// www.pap-thecoastcentre.org/razno/Protocole\%20GIZC\%20Formatte.pdf

5. Yves Hénocque et Raphaël Billé (2005) présentent la France comme un cas typique d'indocilité à l'égard de la gestion intégrée des zones côtières.

6. On pense notamment à la Coastal Zone Canada Association (CZCA) qui a été créée en 1993 pour parrainer les conférences organisées autour de l'approche GIZC.

7. Comme nous l'avons précisé précédemment, la référence à une gestion intégrée du littoral s'exprime dès les années 1970 à l'échelle nationale avec le Coastal Zone Management Act (1972) ou encore le rapport Piquart (1973) mais aussi au niveau international avec les recommandations respectives du Conseil de l'Europe (1973) et celle de l'OCDE (1976).

8. Sur cette question et en lien avec le domaine des zones côtières, voir la Convention pour la protection de la mer Méditerranée contre la pollution du 16 février 1976. La question de la gestion des espaces marins y est abordée sous l'angle des pollutions et des menaces que ces 
pollutions font peser sur la santé des hommes, les ressources biologiques et les activités marines. http://195.97.36.231/dbases/webdocs/BCP/bc76_fre.pdf

9. Edgard Morin parle à ce sujet de «corrélation antagoniste » entre le développement de la société industrielle et les critiques qui l'accompagnent.

10. On notera qu'il y a toujours eu des initiatives de protection de la nature dans le monde moderne, notamment pour répondre aux dégradations liées à l'industrialisation et l'urbanisation croissantes. Toutefois, l'activité de protection n'est tolérée qu'à la marge de l'entreprise industrielle, autrement dit, tant qu'elle n'empiète pas sur l'entreprise productiviste. Ainsi, la protection de la nature ne constitue que l'arrière-plan du cadre quasi absolu à travers lequel l'homme pense son action c'est-à-dire le processus industrialo-capitaliste; reléguée à des aires périphériques, elle vient en quelque sorte compenser à la marge les excès de la modernité (Rodary et al., 2003).

11. Rachel Carson, Silent Spring (1962). Devenu un véritable best-seller mondial, ce livre fait un état des lieux des dommages causés par les pesticides agricoles et à travers cela dénonce toute la machine agro-scientifique qui participe à la production de ces produits toxiques. Cet ouvrage fait de la question des pesticides l'un des enjeux majeurs à travers lesquels les gens ont compris ce qu'était la crise environnementale.

12. A partir d'une simulation du futur via un modèle informatisé innovant et d'une approche systémique, ce rapport présente une série de scénarii inquiétants sur les relations entre croissance économique et dégradation de l'environnement.

13. L'écologie politique des années 1970 se trouve très influencée par les analyses de la domination et se présente généralement sous la forme de l'essai. Depuis le tournant des années 1980/1990 et dans le domaine académique anglo-saxon notamment, l'écologie politique renvoie à l'ensemble des travaux spécialisés sur les aspects politiques afférents aux discours et aux pratiques environnementalistes (Greenger et Park, 1994)

14. Philippe Le Prestre (2005: XIV) propose une définition de l'écopolitique internationale comme faisant « référence à l'ensemble des dimensions politiques de l'identification et de la résolution des questions environnementales et, plus particulièrement, aux tentatives des acteurs internationaux d'imposer leurs visions de la sécurité face à la nature ».

$15 . \quad$ http://www.unep.org/Documents.Multilingual/Default.asp? DocumentID $=97 \&$ ArticleID $=1503 \& \mathrm{l}=\mathrm{fr}$

\section{RÉSUMÉS}

Le discours sur la "gestion intégrée des zones côtières" (GIZC) est devenu aujourd'hui incontournable pour les politiques de gestion du littoral. A travers une mise en perspective de l'approche GIZC autour de l'évolution de la pensée écologiste depuis les années 1970, ce papier invite à porter un regard différent sur son émergence politique. Après avoir montré quels sont les mécanismes généralement retenus par la littérature pour dépeindre le processus de politisation de ce discours, il souligne l'importance de tenir compte du cadre cognitif général dans lequel il s'insère. Ainsi, la politisation de l'approche GIZC s'explique en partie par la reconnaissance sociale du développement durable, laquelle rompt avec l'écologisme critique dominant des années 1970. 
Nowadays, the discourse on "integrated coastal zone management" (ICZM) is essential in coastal management policies. Through a comprehension of the evolution of the environmental discourse since the 1970s, this paper offers a fresh perspective to understand the politicization of the ICZM approach. After showing what mechanisms are generally put forward in the literature to depict this politicization, it emphasizes the importance considering its overall cognitive framework. Thus, politicization of ICZM discourse is partly linked with the social recognition of sustainable development thought, which breaks with the dominant critical environmentalism of the 1970s.

\section{INDEX}

Mots-clés : gestion intégrée des zones côtières, écologie, politisation, développement durable, discours

Keywords : integrated coastal zone management, ecology, politicization, sustainable development, discourse

\section{AUTEUR}

\section{CÉLINE SÉGALINI}

Céline Ségalini est doctorante, rattachée au laboratoire « Les Afriques dans le Monde » (Sciences Po Bordeaux) et financée par le bureau d'études SOFRECO dans le cadre d'une thèse CIFRE. Ses travaux portent sur l'articulation entre les dynamiques professionnelles et la réforme de l'action publique engagée dans le cadre d'un programme de « gestion intégrée des ressources marines et côtières » au Sénégal. celine.segalini@yahoo.com 